\title{
Eating breakfast daily matters for students
}

\section{Opinion}

Students require eating varieties of foods from all food groups (milk, fruits, vegetables, grains, meat, and healthy oil) in proper amounts to ensure adequate growth and development. Proper nutrition helps students grow properly, improves their immunity against infection, and allows them to be more alert in school. Poor nutrition increases their risks for obesity or malnutrition, diabetes, weak bones, anemia, and heart disease. It also affects their education performance, learning abilities, activity levels, and growth patterns.

Eating a healthy, well-balanced breakfast is important for weight control and brain development, especially for children and adolescents. Research shows that consuming a nutritious, wholesome breakfast at the start of the day supports children's learning abilities, attention, and memory span. Some healthy breakfast items include:

i. Whole grains or unsweetened cereals, rather than refined grains or white flour. Whole grains are rich in fiber and other nutrients, which increase satiety and stabilize blood sugar.

ii. Low-fat or non-fat milk and its products, which are good sources of protein, calcium, and Vitamin D. Low-fat and nonfat milk products have fewer calories and fat.

iii. Protein-rich foods, such as eggs, low-fat cheese, low-fat yogurt, and peanut butter. Cream cheese and fried eggs should be avoided.

iv. Fresh or dried fruits or unsweetened juice. Fruits are an excellent source of vitamins, fiber, and antioxidants, which aid in children's development and immunity.
Volume 2 Issue 5- 2015

\author{
Mireille Najiar \\ Tufts University, USA \\ Correspondence: Jianjun Mireille Najjar, Nutrition \\ Communication graduate student at The Friedman School, Tufts \\ University, USA, Email mireillenaj@gmail.com
}

Received: July 9, 2015 | Published: September 15, 2015

Brain function and the ability of students to focus and to do well at school depend on the foods they eat. Students who eat healthier, low-fat breakfast to salty, sugary snacks and drinks will have more balanced energy levels and improved concentration, which ultimately leads to better performance in school. Avoiding unhealthy breakfast, such as waffles and pancakes with extra syrup, sweetened cereals, sausages, and white bread, may reduce the risk of certain health problems. Eating the right foods provides the key to a healthier life, both now and for students' futures.

\section{Acknowledgements}

None.

\section{Conflict of interest}

The author declares no conflict of interest. 